\title{
Spine morphogenesis in newborn granule cells is differentially regulated in the outer and middle molecular layers
}

\author{
Chunmei Zhao*, Jessica Jou*, Lisa J Wolff ${ }^{\star}$, Huaiyu Sun\#, and Fred H. Gage* \\ *Laboratory of Genetics, Salk Institute for Biological Studies, La Jolla, CA 92037 \\ \#Molecular and Cell Biology Laboratory, Salk Institute for Biological Studies, La Jolla, CA 92037
}

\begin{abstract}
New neurons are continuously added to the hippocampus of adult mammals. Their survival and integration into the circuitry are highly dependent on experience. Here we show that mushroom spine formation in newborn granule cells was modulated by experience and that dendritic segments in different areas of the molecular layer were differentially regulated. Specifically, spines of new neurons in the outer molecular layer of the dentate gyrus were more readily influenced by non-spatial features in the living environment. Those in the middle molecular layer were more likely to be influenced by the size of the living environment. Therefore, the activity of cortical inputs into newborn granule cells may be reflected in the formation of mushroom spines in different dendritic segments in the molecular layer.
\end{abstract}

\section{Keywords}

neurogenesis; hippocampus; dendritic spine; mushroom spine; running; enriched environment

\section{Introduction}

New neurons are continuously generated in the dentate gyrus (DG) of the hippocampus throughout life in essentially all mammals examined (Altman and Das, 1965; Eriksson et al., 1998). The integration of newborn granule cells (GC) is regulated by experience. For example, the "experiences" of new neurons during the immature stage shape their integration into the circuitry so that more neurons will survive and express the immediate early gene c-fos or Zif268 in response to the same experiences when they are mature (Tashiro et al., 2007). This phenomenon suggests that some of these new neurons may encode certain features of the "experiences." Indeed, postnatally born GCs are needed for

Corresponding author: Fred H. Gage, Laboratory of Genetics, Salk Institute for Biological Studies, La Jolla, CA 92037, gage@ salk.edu, Tel: 1-858-453-4100 x 1012, Fax: 1-858-597-0824.

Conflict of Interest Statement

The authors have no conflict of interest.

Role of Authors

All authors had full access to all the data in the study and take responsibility for the integrity of the data and the accuracy of the data analysis. Study concept and design: CZ and FHG. Acquisition of data: CZ, JJ and LJW. Analysis and interpretation of data: CZ and HS. Drafting of the manuscript: CZ. Obtained funding: FHG. 
the accurate recall of previously learned spatial and contextual memories (Arruda-Carvalho et al., 2011).

The main cortical inputs to the DG arrive from the perforant path axons that originate from layer II of the entorhinal cortex (EC). The outer and middle molecular layers of the DG receive inputs from the lateral and medial ECs, respectively (O'Keefe, 1978; Steward, 1976). Neurons in the medial and lateral ECs respond to different features in the environment. Grid cells, boundary cells and head-direction cells reside in the medial EC and provide spatial information (i.e., where) to the hippocampus. In contrast, neurons in the lateral EC respond to stimuli such as odor and objects and provide non-spatial information (i.e., what) to the hippocampus (Manns and Eichenbaum, 2006; Moser et al., 2008).

Perforant path axons synapse onto the dendritic spines of GCs. Because dendritic spines are the major postsynaptic sites of excitatory synapses, the integration of newborn GCs can be correlated with spine growth and maturation. The peak of spine growth occurs when newborn GCs are 2-4 weeks old, when these cells have a low threshold for long-term potentiation (Ge et al., 2007; Schmidt-Hieber et al., 2004; Zhao et al., 2006). During this time window, a large portion of the synapses that are formed on newborn GCs are on multiple synapse boutons (MSBs) (Toni et al., 2007). After an extended period of maturation, the numbers of mushroom spines increase, synapses on MSBs decrease and newborn GCs become indistinguishable from existing cells in their electrophysiological properties (Laplagne et al., 2006; Toni et al., 2007; Zhao et al., 2006).

To determine how cortical inputs into the DG shape the integration of adult-born GCs, we used a series of different animal holding cages so that the complexity and/or the size of the cage were different than the standard static mouse cage. We then used spine morphogenesis to assess the integration of adult-born GCs. We found that mushroom spine formation in the outer and middle molecular layers was differentially regulated by changes in the living environment.

\section{Materials and Methods}

\section{Mice}

Six- to eight-week-old C57B1/6 female mice were used for all studies. Six different cage conditions were used throughout the study, including a static mouse sedentary cage (S, $\left.28 \times 17 \mathrm{~cm}^{2}\right)$, ventilated mouse sedentary cage $\left(\mathrm{V}, 28 \times 17 \mathrm{~cm}^{2}\right)$, standard rat sedentary cage $\left(\mathrm{R}, 43 \times 21.5 \mathrm{~cm}^{2}\right.$ ), standard rat cage with running wheel $\left(\mathrm{RW}, 43 \times 21.5 \mathrm{~cm}^{2}\right.$ ), mouse single activity wheel chamber (Lafayette Instruments) $\left(\mathrm{CW}, 30.5 \times 19 \mathrm{~cm}^{2}\right)$, mouse single activity wheel chamber with running wheel removed $\left(\mathrm{C}, 30.5 \times 19 \mathrm{~cm}^{2}\right)$, and custom-designed enriched environment cage (EE, $91.5 \times 91.5 \mathrm{~cm}^{2}$ ). All mice were group housed (4 in each cage for S, V, R, RW, CW, C and 8/cage for EE). The animal protocols were all approved by the Salk Institutional Animal Care and Use Committee.

\section{Retrovirus-mediated labeling of newborn GCs}

Newborn GCs were labeled with retrovirus-mediated expression of green fluorescent protein as previously described (Tashiro et al., 2006; Zhao et al., 2006). Briefly, $1 \mu \mathrm{l}$ of retrovirus 
CAG-GFP at $1-5 \times 10^{8} / \mathrm{ml}$ was infused into the right hemisphere using the following coordinates in reference to Bregma: anterioposterior: $-1.7 \mathrm{~mm}$, lateral: $-1.6 \mathrm{~mm}$, dorsal/ ventral: -2 from dura). All mice were sacrificed at 8 weeks after virus injection for morphological analyses. In some experiments, mice were given 2 doses of $\mathrm{BrdU}(50 \mathrm{mg} / \mathrm{kg}$, 6 hours apart) the day before virus injection for a more accurate assessment of the level of neurogenesis.

\section{Immunohistochemistry and cell quantification}

Mice were given a lethal dose of anesthesia (Ketamine $100 \mathrm{mg} / \mathrm{kg}$, Xylazine $10 \mathrm{mg} / \mathrm{kg}$ ) and perfused transcardially with $0.9 \% \mathrm{NaCl}$ followed by $4 \%$ para formaldehyde (PFA). Mouse brains were post fixed in 4\% PFA overnight and transferred to a $30 \%$ sucrose solution for 48 hours. Coronal sections of $40 \mu \mathrm{m}$ thickness were prepared with a sliding microtome. Brain sections of one-in-six series were selected for immunostaining (for BrdU cell counting, rat anti-BrdU, 1:100, Acurate) or DAPI staining. The characterization of rat anti-BrdU has been previously documented (Mathews et al., 2010). BrdU+ or GFP+ cells in the GC layer were visualized and counted with a Nikon E800 microscope (Melville, NY). The total number of labeled cells per DG was estimated by multiplying the number counted from the one-in-six series by 6 .

\section{Confocal imaging and spine analysis}

All images were acquired through the Bio-Rad R2100 confocal system or the Zeiss710 confocal system. For unbiased sampling of newborn GCs, the labeling efficiency of an individual mouse was first visually inspected under an epifluorescent microscope. A number " $n$ " was then decided based on the labeling efficiency so that every $n^{\text {th }}$ GFP+ cell would be selected providing that this cell contained at least one dendrite that remained intact from the cell body to the outer third of the molecular layer. If the $\mathrm{n}^{\text {th }}$ cell did not fit the criteria, the next cell that met the criteria would be selected. The sampling started from the first anterior section that displayed GFP+ GCs, and all sections across the anteroposterior axis were sampled. All mice were numerically coded and the investigators were blind to the treatment conditions of individual animals until all images were acquired and quantified. Images of the overview of BrdU labeling were taken with a $20 \times$ objective (Bio-Rad R2100) or a $25 \times \mathrm{W}$ objective (Zeiss710). Images for assessment of the whole cell morphology were obtained with a 40× oil objective (numerical aperture, NA 1.3, Nikon). For spine analyses, dendritic processes of GFP+ cells in the outer and middle third of the molecular layer were imaged with a $60 \times$ oil objective (NA 1.4, Nikon, on Bio-Rad R2100) or with a 63×oil objective (NA 1.4, Zeiss, on Zeiss710), respectively (Fig 1A-C). Each granule cell was represented by a single dendritic segment in the middle or outer molecular layer, which was achieved by imaging only the dendritic segments that were still visually connected to the cell body after tissue sectioning. Images in the outer and middle molecular layers were acquired at different times and on different systems. Therefore, the absolute numbers in different figure panels cannot be cross-compared. The raw confocal image files were subjected to 10 iterations of deconvulution (AutoDeblur, AutoQuant, Troy, NY). Dendrite measuring and spine analyses have been described in detail (Zhao et al., 2006). Briefly, the center of the dendritic shaft was manually traced using the IGL Trace program. For categorizing mushroom spines, the lengths of the long and short axes of the spine head were obtained by manual tracing and the 
estimated surface area was deduced using the function $0.25 * \pi * d x * d y$ (Fig 1D). Spines with an estimated surface area of $0.4 \mu \mathrm{m}^{2}$ or greater were considered to be mushroom spines (Fig 1D, the sizes of spines with major and minor axes marked from left to right were $0.24,0.36$ and $0.41 \mu \mathrm{m}^{2}$ respectively). The cut-off value of $0.4 \mu \mathrm{m}^{2}$ was the nearest round-up of the value of mean $+2 *$ standard deviation of the surface area of all spines from 3 independent confocal images (Zhao et al., 2006). Spines with an area between 0.25 and $0.35 \mu \mathrm{m}^{2}$ were most frequently analyzed. Relative differences between groups remained consistent when the cut-off value was set at $10 \%$ above or below $0.4 \mu \mathrm{m}^{2}$. Due to the nature of manual spine analyses, a single experimenter quantified each set of experiments.

\section{Imaging Processing}

Examples of spine images were cropped to the same size and assembled with Adobe Photoshop without further adjustments. Images of BrdU immunohistochemistry and GFPlabeled newborn GCs were adjusted with contrasting in Adobe Photoshop for better visualization. All figures were assembled in Adobe Illustrator and exported in tiff format.

\section{Statistical analysis}

All data were presented as mean \pm standard error. Statistical comparisons were done using ANOVA or unpaired two-tailed t-test. The Bonferroni's Multiple Comparison test was used for post-test comparisons. The n number represents all of the dendritic segments analyzed within each group (3-4 mice each group). Because a single dendritic segment represented each granule cell, the n number also represents all of the neurons analyzed within each group.

\section{Results}

We previously reported that running promoted mushroom spine formation in newborn GCs (Zhao et al., 2006). This finding was based on a comparison between mice that were housed in a standard mouse static cage (Fig 2A, S) and mice housed in a mouse single activitywheel chamber (Fig 2B, CW). In addition to the running wheel, the $\mathrm{CW}$ cages had some other features that were different from $\mathrm{S}$ cages. The $\mathrm{CW}$ cages were slightly larger than $\mathrm{S}$ cages. The $\mathrm{CW}$ cages also contained more objects: in addition to the feeder and water bottle, they contained a special apparatus that held the running wheel in position. Therefore, the $\mathrm{CW}$ cages were more complex, both spatially and non-spatially. Non-spatial and spatial information may be relayed into the DG from lateral and medial ECs, respectively. Since lateral and medial ECs project to outer and middle molecular layers of the DG, respectively, we sought to determine whether dendritic segments of newborn GCs could be differentially regulated by spatial and non-spatial changes in the living environment.

\section{Mushroom spine formation in different dendritic segments of newborn GCs was modulated by housing conditions}

To confirm our previous findings, we repeated the experiment with the $\mathrm{S}$ and $\mathrm{CW}$ cages. We also added a different running wheel cage: a standard rat cage equipped with 2 running wheels (Fig 2C, RW). The rat cage provided a much larger space (inside parameter: $43 \times 21.5$ $\mathrm{cm}^{2}$ for rat cage, $30.5 \times 19 \mathrm{~cm}^{2}$ for $\mathrm{CW}$ cage and $28 \times 17 \mathrm{~cm}^{2}$ for $\mathrm{S}$ cage). We followed the 
same experimental paradigm as that used in our previous study (Zhao et al., 2006). Female C56Bl/6 mice were group housed in these different experimental cages 1 week before virus injection and BrdU administration, and the mice were sacrificed 8 weeks after the virus injection.

First, we validated that the running wheel housing in both cages ( $\mathrm{CW}$ and RW) promoted neurogenesis; there was an increase in the number of BrdU+ cells in the DG of mice housed in the CW and RW cages (Fig 2D-G, F(2,9)=6.994, $\mathrm{p}<0.05$, $\mathrm{n}=4$ mice for each group, $\mathrm{S}$ : 699 \pm 103 , CW: 2373 \pm 710 , RW: 3132 \pm 387 ). Bonferroni's Multiple Comparison test detected a significant difference in BrdU cell numbers between $\mathrm{S}$ and RW.

Newborn GCs from different groups were similar in overall morphology by visual inspection (Fig H-J). These cells did not differ significantly in total dendritic length (Fig $2 \mathrm{~K}, \mathrm{~F}(2,69)=2.129, \mathrm{p}=0.13 ; \mathrm{S}: 608.2 \pm 35.7, \mathrm{n}=29 ; \mathrm{CW}: 636.9 \pm 44.7, \mathrm{n}=24$; RW: $724.9 \pm 37.4$ $\mu \mathrm{m}, \mathrm{n}=19)$. Newborn GCs from the RW group had more branching points than those from the $S$ group (Fig $2 L, F(2,69)=3.893$, p<0.05; S: 5.14 \pm 0.30 , n=29; CW: 6.08 \pm 0.53 , n=24; RW: $6.84 \pm 47, \mathrm{n}=19)$.

In the outer molecular layer, total spine density was similar in all conditions (Fig $2 \mathrm{M}-\mathrm{N}$, $\mathrm{F}(2,134)=0.009, \mathrm{p}>0.05, \mathrm{~S}: 3.07 \pm 0.12, \mathrm{n}=34 ; \mathrm{CW}: 3.07 \pm 0.10, \mathrm{n}=55 ; \mathrm{RW}: 3.05 \pm 0.10$ per $\mu \mathrm{m}$, $\mathrm{n}=48$ ). Mushroom spine density was significantly higher in samples from both the $\mathrm{CW}$ and RW cages (Fig 2O, F(2,134)=11.51, p<0.01, S: 0.070 .011 , n=34; CW: 0.181 $\pm 0.018, n=55$; RW: $0.162 \pm 0.016$ per $\mu \mathrm{m}, \mathrm{n}=48$ ). In the middle molecular layer, total spine density was not influenced by housing conditions either (Fig 2P-Q, Fig 2J, F(2,134)=2.429, p>0.05, S: $3.05 \pm 0.16, n=38$; CW: $2.96 \pm 0.10, n=54$; RW: $2.67 \pm 0.08$ per $\mu \mathrm{m}, \mathrm{n}=45$ ). Mushroom spine density was higher in the middle molecular layer in samples from RW cages and was modestly increased in those from CW cages (Fig $2 \mathrm{R}, \mathrm{F}(2,134)=6.235$, $\mathrm{p}<0.01, \mathrm{~S}$ : 0.041 $\pm 0.006, \mathrm{n}=38$; CW: $0.064 \pm 0.008, \mathrm{n}=54$; RW: $0.083 \pm 0.009$ per $\mu \mathrm{m}, \mathrm{n}=45$ ).

To determine whether these data may be influenced by the manual tracing of spine heads or the utilization of different confocal systems, we did additional analyses on the data sets in Fig 2 R. First, the threshold for mushroom spine was set at $\pm 10 \%$ of $0.40 \mu \mathrm{m}^{2}$, specifically at 0.36 or $0.44 \mu \mathrm{m}^{2}$, and mushroom spine density was recalculated for each dendrite. As expected, a cut-off value of $0.36 \mu \mathrm{m}^{2}$ yielded more mushrooms spines, whereas a cut-off value of $0.44 \mu \mathrm{m}^{2}$ identified fewer mushroom spines (Fig $3 \mathrm{~A} \& \mathrm{~B}$ ). However, mushroom spine density was significantly higher in RW group than that in $\mathrm{S}$ group in both cases. This is consistent with the previous observation when spines of $0.40 \mu \mathrm{m}^{2}$ or larger were considered to be mushroom spines (Fig 3A, F(2,134)=7.933, p<0.01; Fig 3B, $\mathrm{F}(2,134)=5.554, \mathrm{p}<0.01)$. In addition, a distribution histogram of all traced spines showed that spines of $0.25-0.35 \mu \mathrm{m}^{2}$ were most frequently analyzed (Fig 3C). These data suggest that manual image analysis by different individuals or slight variations in microscope calibration should not obscure the comparison between study groups, and that spines $0.35 \mu \mathrm{m}^{2}$ or larger are likely all included in the analysis.

Therefore, mushroom spine formation in the outer molecular layer was enhanced in mice housed in both running wheel cages, while mushroom spine formation in the middle 
molecular layer was enhanced only in mice housed in the RW cage. These data suggest that mushroom spine formation in the outer and middle molecular layers can be differentially regulated by experience.

\section{Differential regulation of cell genesis and spine maturation}

Since both CW and RW cages display additional differences from the control S cage beside the running wheel(s), we could not determine whether the enhanced spine maturation in the $\mathrm{CW}$ and RW groups was promoted by exercise. Therefore, we set up 2 experiments to use similar cages as controls. To control for running in the $\mathrm{CW}$ cage, we used the $\mathrm{C}$ cage, which was essentially the same as the CW cage except that the running wheel was removed (Fig 4A, B). Two groups of mice were acclimated in the $\mathrm{C}$ and $\mathrm{CW}$ cages for 7 days before virus injection/BrdU administration and the mice were sacrificed 8 weeks later. Quantification of BrdU cell number confirmed that inclusion of a running wheel in the cage indeed promoted neurogenesis (Fig 4C,D). Total BrdU number in the DG of the hemisphere contra-lateral to the virus injection side was increased nearly 3 -fold in the $\mathrm{CW}$ cage compared to the $\mathrm{C}$ cage (C: $867 \pm 118, \mathrm{n}=4 ; \mathrm{CW}: 3267 \pm 589, \mathrm{n}=4 ; \mathrm{p}<0.01$ ). There was no difference in total spine density (Fig 4E-F, C: $1.87 \pm 0.09$, n=23; CW: $1.87 \pm 0.06$ per $\mu \mathrm{m}, \mathrm{n}=25$ ) or mushroom spine density (Fig 4G, C: $0.130 \pm 0.018, n=23$; CW: $0.107 \pm 0.014$ per $\mu \mathrm{m}, \mathrm{n}=25$ ) in the outer molecular layer between the 2 groups. Neither was there a significant change in total spine density (Fig 4H-I, C: $2.76 \pm 0.12$, n=34; CW: $2.52 \pm 0.09$ per $\mu \mathrm{m}, \mathrm{n}=36, \mathrm{p}=0.1$ ) or mushroom spine density (Fig 4J, C: $0.062 \pm 0.010, n=34$; CW: $0.079 \pm 0.010$ per $\mu \mathrm{m}, \mathrm{n}=36 ; \mathrm{p}=0.23$ ) in the middle molecular layer.

To control for running in the RW cage, we used the standard rat cage without running wheels (Fig 5A, B). These two groups of mice were not administered BrdU, so we quantified GFP+ cell number to examine the level of neurogenesis (Fig 5C, D). The number of GFP-labeled new neurons was higher in the mice housed in the RW cage than in the mice housed in the R cage, confirming the effect of running in promoting cell genesis (R: 195 \pm 67 , $\mathrm{n}=4$; RW: $1305 \pm 501, \mathrm{n}=4 ; \mathrm{p}=0.07$ ). Due to variations in GFP labeling, the difference in cell number did not reach statistical significance. In the outer molecular layer, there was no difference in total spine density (Fig 5E-F, R: $3.29 \pm 0.24$, n=20; RW: $3.05 \pm 0.17$, n=30 per $\mu \mathrm{m} ; \mathrm{p}=0.40$ ). However, mushroom spine density was higher in the RW group (Fig $5 \mathrm{G}, \mathrm{R}$ : $0.025 \pm 0.009, n=20 ; R W: 0.072 \pm 0.014$ per $\mu \mathrm{m}, \mathrm{n}=30 ; \mathrm{p}<0.05$ ). Strikingly, in the middle molecular layer, the RW group did not differ from the R group in either total spine density (Fig 5H-I, R: 3.07 $\pm 0.19, \mathrm{n}=26$; RW: $2.81 \pm 0.14$ per $\mu \mathrm{m}, \mathrm{n}=34 ; \mathrm{p}=0.26$ ) or mushroom spine density (Fig 5J, R: 0.093 $\pm 0.016, n=26$; RW: $0.093 \pm 0.012$ per $\mu \mathrm{m}, \mathrm{n}=34$ ). In this experiment, inclusion of running wheels promoted mushroom spine formation in the outer but not in the middle molecular layer.

Therefore, when the size of the living environment was held constant, addition of a running wheel did not affect mushroom spine formation in newborn GCs in the middle molecular layer. However, mushroom spine formation in the outer molecular layer was promoted by inclusion of running wheels in the rat cage. Again, these data indicate that spine maturation in the middle and outer molecular layers is differentially regulated by experience. 


\section{EE promotes mushroom spine formation in both the outer and middle molecular layers}

We next predicted that an EE cage should promote mushroom spine formation in both the outer and the middle molecular layers since it contained more objects (more non-spatial information) and was much bigger in size (more spatial information). To test this hypothesis, we used a custom-designed EE cage that measured $91.5 \times 91.5 \mathrm{~cm}^{2}$ and contained tunnels and domes in addition to a feeder and water bottle (Fig 6B). To specifically test the effect of non-spatial input of the environment without the interference of exercise, we did not place any running wheels in this environment. A group of 8 mice was housed in this environment for the duration of the experiment but only 4 were used for experimental analyses. For the control cage, we used a standard mouse ventilated cage due to the overall cage change in the animal holding room at the Salk Institute (Fig 6A). The only difference between the ventilated cage and the previously used static control cage was that there was a water valve on the backside of the cage. To minimize differences, we still used bottled water and placed the ventilated cage on static racks. Consistent with previous findings, EE housing increased the number of newborn cells (Fig 6C-D, V: 366 \pm 45 , n=4; EE: 602 $\pm 44, n=4 ; p<0.01$ ). EE housing did not affect dendritic growth. We did not observe a significant difference in total dendritic length (V: $567 \pm 27, n=30$; EE: $569 \pm 23 \mu \mathrm{m}, \mathrm{n}=35$ ) or dendritic branching points (V: $5.50 \pm 0.31, \mathrm{n}=30 ; \mathrm{EE}: 5.71 \pm 0.28, \mathrm{n}=35)$. Total spine density was not affected in either the outer (Fig 6E-F, V: 3.06 \pm 0.15 , n=39; EE: $3.04 \pm 0.17$ per $\mu \mathrm{m}, \mathrm{n}=27$ ) or middle molecular layer (Fig 6H-I, V: $2.80 \pm 0.14$, n=31; EE: $2.87 \pm 0.12$ per $\mu \mathrm{m}, \mathrm{n}=32$ ). In contrast, mushroom spine density was increased in both the outer (Fig 6G, V: 0.095 \pm 0.013 , n=39; EE: $0.157 \pm 0.023$ per $\mu \mathrm{m}, \mathrm{n}=27 ; \mathrm{p}<0.05$ ) and middle (Fig 6J, V: 0.042 $\pm 0.008, \mathrm{n}=31$; EE: $0.089 \pm 0.013$ per $\mu \mathrm{m}, \mathrm{n}=32 ; \mathrm{p}<0.01)$ molecular layer. Therefore, increasing both the nonspatial and spatial complexity of the living environment promoted mushroom spine formation in both the outer and middle molecular layers.

\section{Discussion}

In this study, we examined the integration of newborn GCs under different housing conditions. We found that the presence of a running wheel consistently promoted neurogenesis, whereas its effect on spine morphogenesis was layer specific and dependent on the non-spatial and spatial complexity of the living environment.

\section{Larger space promoted spine maturation in the middle molecular layer}

In the middle molecular layer, mushroom spine formation in newborn GCs was promoted only when the size of the living environment was significantly larger. Mushroom spine density was higher in the RW cage compared to the static mouse cage (Fig 2R) and higher in the EE cage compared to the ventilated mouse cage (Fig 5J). Both RW and EE cages were much larger than mouse cages (RW: $925 \mathrm{~cm}^{2}$, EE: $8372 \mathrm{~cm}^{2}$, S or V: $476 \mathrm{~cm}^{2}$ ). When the size of the cages was held constant, the addition of running wheels to the cage did not have any effect on mushroom spine formation in the middle molecular layer (Fig 3J, CW vs C and Fig 4J, RW vs R). Therefore, our data suggest that spine maturation in the middle molecular layer was specifically regulated by the spatial component of the living environment. This finding is consistent with the fact that the middle molecular layer of the 
DG receives input from the medial EC, which mainly provides spatial information to the hippocampus.

\section{Non-spatial components in the living environment promoted mushroom spine formation in the outer molecular layer}

Spine maturation of newborn GCs in the outer molecular layer appeared to be more readily influenced in our experiments. We observed an increase in mushroom spine formation in the outer molecular layer in all but one experiment. In Figure 3G, when the CW was compared to the $\mathrm{C}$ group, there was no difference in mushroom spine density in the outer molecular layer. However, when the RW was compared to the R group, we observed an increase in mushroom spine density in the outer molecular layer (Fig 4G). It is unlikely that exercise was the major contributor to spine maturation because adding a running wheel to the appropriate control cages did not always promote mushroom spine formation (Fig 3G, comparing $\mathrm{CW}$ to $\mathrm{C}$ ). Furthermore, housing mice in an enriched environment with no running wheel also promoted mushroom spine formation in the outer molecular layer (Fig $5 \mathrm{G})$. Therefore, we speculate that the running wheels in the RW cage served as an interesting object and added more non-spatial content to the rat cage (Fig 4A-B). In the case of the $\mathrm{C}$ cage, because the cage already had more non-spatial complexity compared to the standard mouse or rat cages, adding a running wheel may not have had the same level of impact as it would in a simpler environment.

\section{Differential regulation of neurogenesis and neuronal integration}

Our data suggested that the level of neurogenesis and the integration of newborn cells could be regulated differentially. Running consistently promoted neurogenesis in all experiments but did not have a significant effect on neuronal maturation. It only increased mushroom spine formation in the outer molecular layer in the RW compared to R group, and it did not affect mushroom spine formation in the middle molecular layer when same-size cages were used as controls. In contrast, mushroom spine formation in newborn GCs was more readily influenced by the spatial and non-spatial complexity of the living environment. Interestingly, spatial learning shapes both the selection and connectivity of newborn cells (Dupret et al., 2007; Tronel et al., 2010). Furthermore, adult-born neurons have been found to contribute to long-term spatial memory in the Morris water maze task (Deng et al., 2009; Snyder et al., 2005). Thus, our data partially explain how running is beneficial to hippocampus-dependent learning and memory: running promotes the generation of adult-born GCs that are readily recruited to experiences such as spatial exploration.

\section{Dendritic growth or spine growth in general was not significantly influenced by EE}

Throughout our studies, we observed an increase in branching points only when animals were housed in the RW cage (compared to the static mouse cage). In general, no difference was detected in dendritic growth, branching points or total spine density. Similar to our findings, Piatti et al. (2011) found that running could promote spine growth in neurons in the temporal DG at 21 days post injection and that spine density did not differ between neurons found in the septal and temporal DG at $56 \mathrm{dpi}$ in mice. In addition, electrical stimulation of EC did not affect dendritic or spine growth, although it efficiently increased the total number of newborn cells in mice (Stone et al., 2011). However, Abrous and colleagues have 
consistently reported that spatial learning promoted dendritic growth and branching and increased total spine density in adult-born GCs (Lemaire et al., 2012; Tronel et al., 2010). Aside from the difference in the stimuli used in these studies (exercise, EE and spatial learning), a possible explanation for this discrepancy is that we may have underestimated the effects of running and EE. All mice were group housed in this study and did not have the same access to running wheels. More importantly, only a portion of newborn cells would survive the first 4 weeks during their development, and running/EE housing was able to rescue the cells that would have died if the mice were housed in standard conditions (Kempermann et al., 2003; Kempermann et al., 1997; Muotri et al., 2009; Tashiro et al., 2007). It is possible that the rescued cells were destined to die because they were deficient in dendritic and spine growth. In fact, in a separate study we observed that the average spine density significantly decreased when we forced the survival of newborn cells by overexpressing the pro-survival protein Bcl2 (data not shown). In contrast, when the death of newborn cells was prevented by running or EE in this study, we did not see a decrease in total spine density. Therefore, it is reasonable to speculate that running or EE may increase the survival of newborn cells by enhancing their integration into the system through promoting dendritic and spine growth.

Even with this possible underestimation, we observed that experiences modulated spine maturation in a layer-specific manner that was likely an activity-dependent response with a certain level of spatial resolution. Indeed, activity-dependent structural remodeling can be input-specific at the level of individual spines (Matsuzaki et al., 2004). We would like to propose that input activity from different cortical areas is reflected in layer-specific spine maturation in the dendritic segments of adult-born GCs.

\section{Acknowledgments}

We are grateful to Mary Lynn Gage for editorial comments, Yangling Mu and Star Lee for critical comments, Eunice Mejia and Ilene Chen for help with histology and confocal imaging, and Wei Deng, Brad Aimone and Dane Clemenson for discussions. We also thank James Fitzpatrick, Jamie Kasuboski and Yury Sigal at the Waitt Advanced Biophotonics Center for technical support. This work was supported by the McDonnell Foundation, the JPB Foundation, the Ellison Foundation and, Grant from NIH to FHG.

This work was supported by the U.S. National Institutes of Health (NS-05050217 and NS-05052842), the James s. McDonnell Foundation, the JPB Foundation, and the Ellison Foundation.

\section{References}

Altman J, Das GD. Autoradiographic and histological evidence of postnatal hippocampal neurogenesis in rats. J Comp Neurol. 1965; 124(3):319-335. [PubMed: 5861717]

Arruda-Carvalho M, Sakaguchi M, Akers KG, Josselyn SA, Frankland PW. Posttraining ablation of adult-generated neurons degrades previously acquired memories. J Neurosci. 2011; 31(42):1511315127. [PubMed: 22016545]

Deng W, Saxe MD, Gallina IS, Gage FH. Adult-born hippocampal dentate granule cells undergoing maturation modulate learning and memory in the brain. J Neurosci. 2009; 29(43):13532-13542. [PubMed: 19864566]

Dupret D, Fabre A, Dobrossy MD, Panatier A, Rodriguez JJ, Lamarque S, Lemaire V, Oliet SH, Piazza PV, Abrous DN. Spatial learning depends on both the addition and removal of new hippocampal neurons. PLoS Biol. 2007; 5(8):e214. [PubMed: 17683201] 
Eriksson PS, Perfilieva E, Bjork-Eriksson T, Alborn AM, Nordborg C, Peterson DA, Gage FH. Neurogenesis in the adult human hippocampus. Nat Med. 1998; 4(11):1313-1317. [PubMed: 9809557]

Ge S, Yang CH, Hsu KS, Ming GL, Song H. A critical period for enhanced synaptic plasticity in newly generated neurons of the adult brain. Neuron. 2007; 54(4):559-566. [PubMed: 17521569]

Kempermann G, Gast D, Kronenberg G, Yamaguchi M, Gage FH. Early determination and long-term persistence of adult-generated new neurons in the hippocampus of mice. Development. 2003; 130(2):391-399. [PubMed: 12466205]

Kempermann G, Kuhn HG, Gage FH. More hippocampal neurons in adult mice living in an enriched environment. Nature. 1997; 386(6624):493-495. [PubMed: 9087407]

Laplagne DA, Esposito MS, Piatti VC, Morgenstern NA, Zhao C, van Praag H, Gage FH, Schinder AF. Functional convergence of neurons generated in the developing and adult hippocampus. PLoS Biol. 2006; 4(12):e409. [PubMed: 17121455]

Lemaire V, Tronel S, Montaron MF, Fabre A, Dugast E, Abrous DN. Long-lasting plasticity of hippocampal adult-born neurons. J Neurosci. 2012; 32(9):3101-3108. [PubMed: 22378883]

Manns JR, Eichenbaum H. Evolution of declarative memory. Hippocampus. 2006; 16(9):795-808. [PubMed: 16881079]

Mathews EA, Morgenstern NA, Piatti VC, Zhao C, Jessberger S, Schinder AF, Gage FH. A distinctive layering pattern of mouse dentate granule cells is generated by developmental and adult neurogenesis. J Comp Neurol. 2010; 518(22):4479-4490. [PubMed: 20886617]

Matsuzaki M, Honkura N, Ellis-Davies GC, Kasai H. Structural basis of long-term potentiation in single dendritic spines. Nature. 2004; 429(6993):761-766. [PubMed: 15190253]

Moser EI, Kropff E, Moser MB. Place cells, grid cells, and the brain's spatial representation system. Annu Rev Neurosci. 2008; 31:69-89. [PubMed: 18284371]

Muotri AR, Zhao C, Marchetto MC, Gage FH. Environmental influence on L1 retrotransposons in the adult hippocampus. Hippocampus. 2009; 19(10):1002-1007. [PubMed: 19771587]

O'Keefe, JaNL. The Hippocampus as a Cognitive Map. Oxford: Oxford University Press; 1978. p. 570

Piatti VC, Davies-Sala MG, Esposito MS, Mongiat LA, Trinchero MF, Schinder AF. The timing for neuronal maturation in the adult hippocampus is modulated by local network activity. $\mathrm{J}$ Neurosci. 2011; 31(21):7715-7728. [PubMed: 21613484]

Schmidt-Hieber C, Jonas P, Bischofberger J. Enhanced synaptic plasticity in newly generated granule cells of the adult hippocampus. Nature. 2004; 429(6988):184-187. [PubMed: 15107864]

Snyder JS, Hong NS, McDonald RJ, Wojtowicz JM. A role for adult neurogenesis in spatial long-term memory. Neuroscience. 2005; 130(4):843-852. [PubMed: 15652983]

Steward O. Topographic organization of the projections from the entorhinal area to the hippocampal formation of the rat. J Comp Neurol. 1976; 167(3):285-314. [PubMed: 1270625]

Stone SS, Teixeira CM, Devito LM, Zaslavsky K, Josselyn SA, Lozano AM, Frankland PW. Stimulation of entorhinal cortex promotes adult neurogenesis and facilitates spatial memory. $\mathrm{J}$ Neurosci. 2011; 31(38):13469-13484. [PubMed: 21940440]

Tashiro A, Makino H, Gage FH. Experience-specific functional modification of the dentate gyrus through adult neurogenesis: a critical period during an immature stage. J Neurosci. 2007; 27(12): 3252-3259. [PubMed: 17376985]

Tashiro A, Zhao C, Gage FH. Retrovirus-mediated single-cell gene knockout technique in adult newborn neurons in vivo. Nat Protoc. 2006; 1(6):3049-3055. [PubMed: 17406567]

Toni N, Teng EM, Bushong EA, Aimone JB, Zhao C, Consiglio A, van Praag H, Martone ME, Ellisman MH, Gage FH. Synapse formation on neurons born in the adult hippocampus. Nat Neurosci. 2007; 10(6):727-734. [PubMed: 17486101]

Tronel S, Fabre A, Charrier V, Oliet SH, Gage FH, Abrous DN. Spatial learning sculpts the dendritic arbor of adult-born hippocampal neurons. Proc Natl Acad Sci U S A. 2010; 107(17):7963-7968. [PubMed: 20375283]

Zhao C, Teng EM, Summers RG Jr, Ming GL, Gage FH. Distinct morphological stages of dentate granule neuron maturation in the adult mouse hippocampus. J Neurosci. 2006; 26(1):3-11. [PubMed: 16399667] 

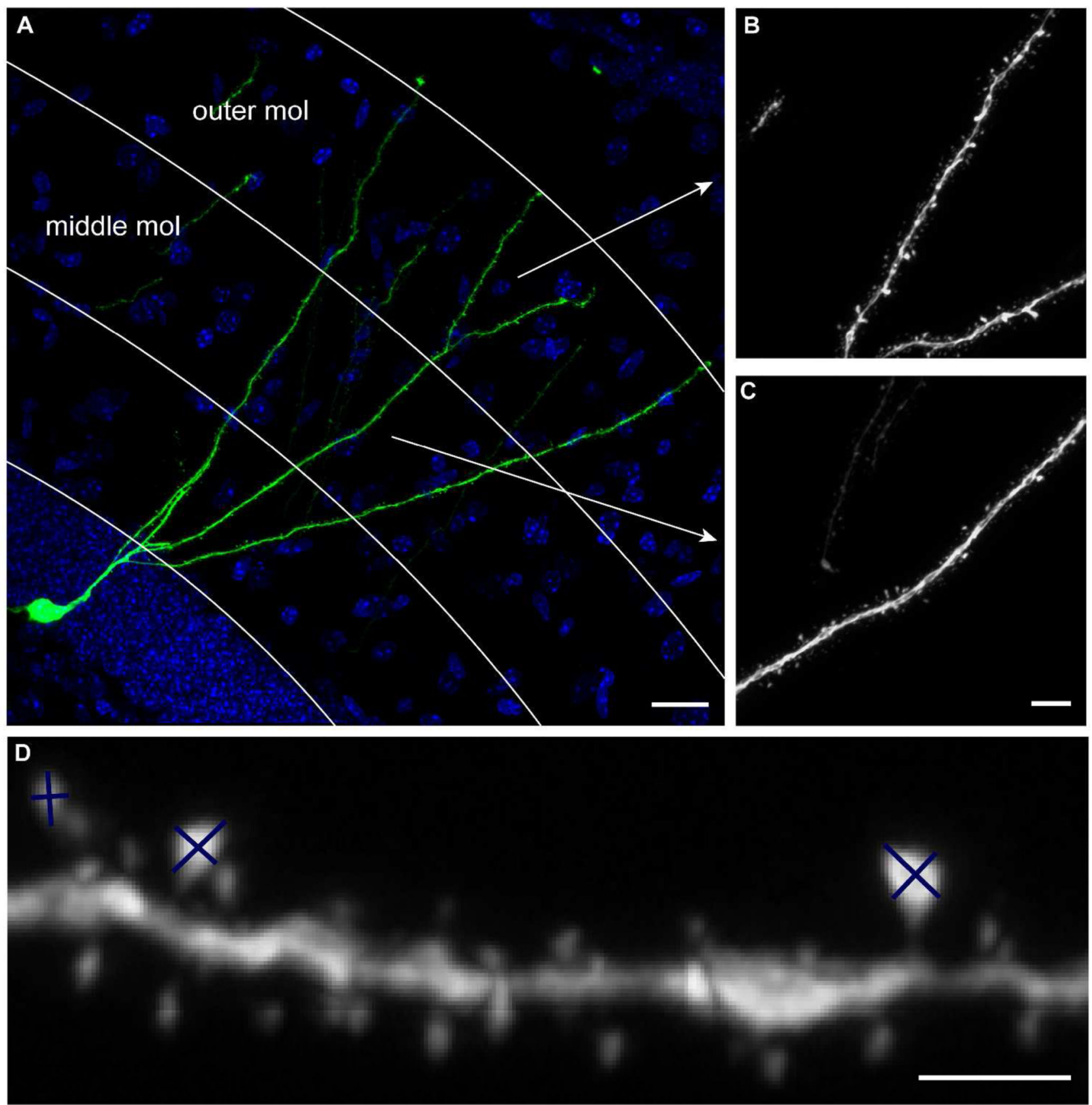

Figure 1. Data acquisition and analyses

(A) Sample image of newborn GC labeled by retrovirus CAG-GFP at 8 weeks post virus injection. Arrows indicate the dendritic processes shown in B and C. (B) Sample image of dendritic processes in the outer molecular layer (mol). (C) Sample image of dendritic processes in the middle molecular layer. (D) Examples of spines whose sizes were estimated by measuring the major and minor axes of the spine heads.. Scale bars $=20 \mu \mathrm{m}(\mathrm{A}), 5 \mu \mathrm{m}$ (B and C), $2 \mu \mathrm{m}(\mathrm{D})$. 
A

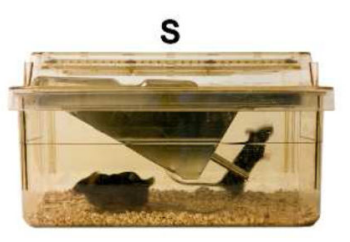

B

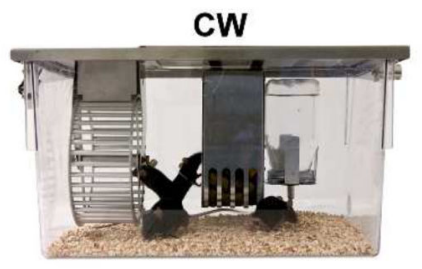

C



D

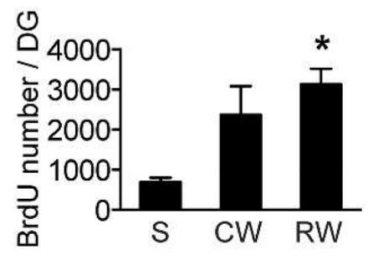

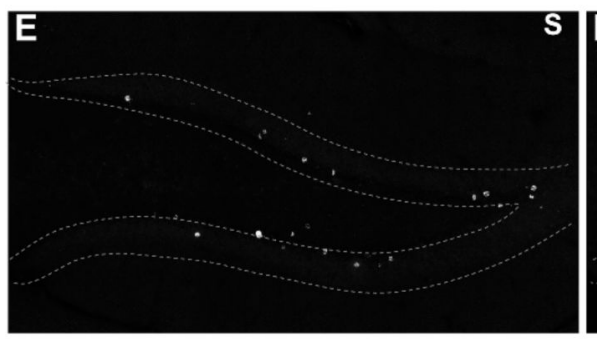
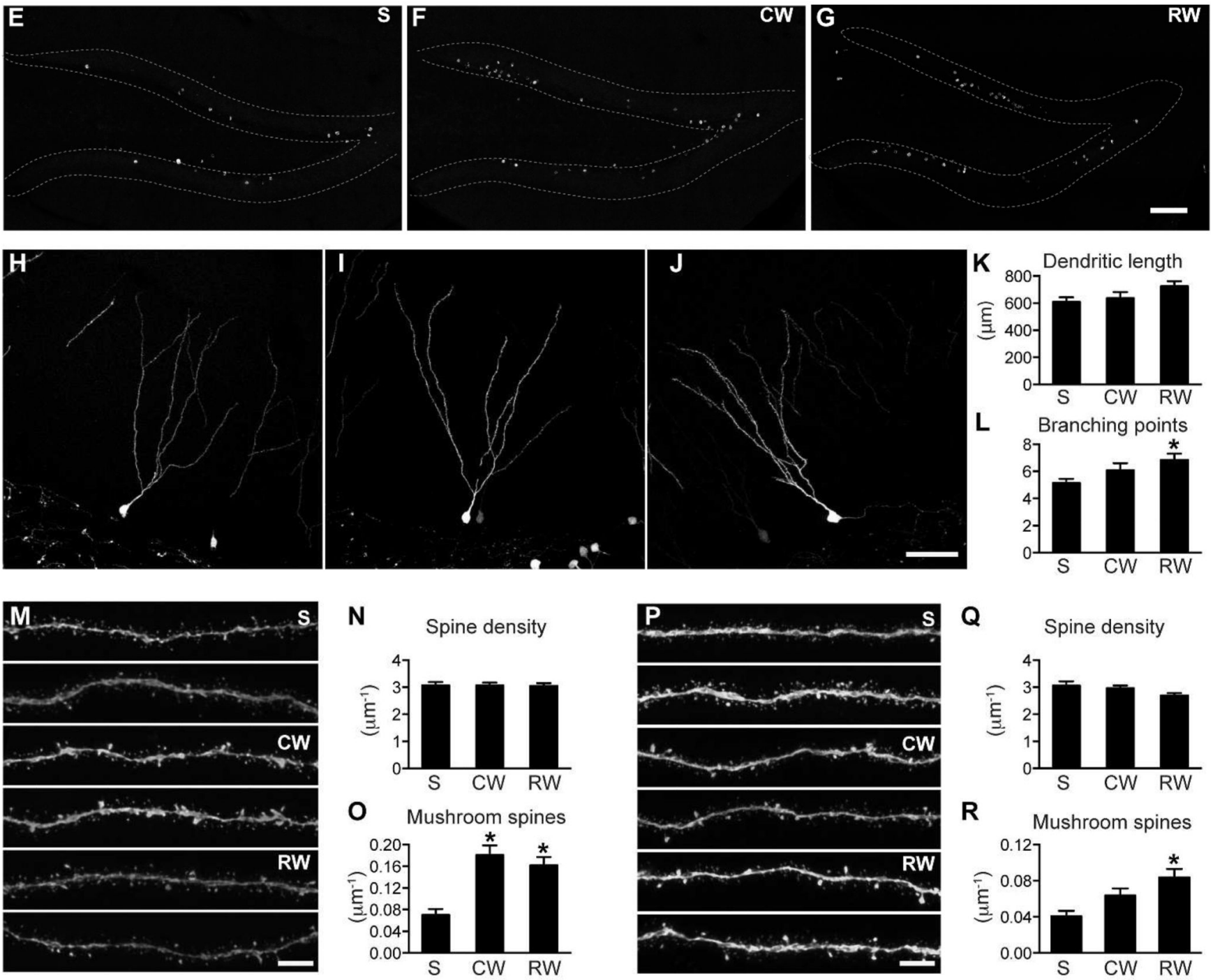

Figure 2. Spine maturation in the outer and middle molecular layers is differentially regulated (A) Standard mouse static cage - S. (B) Mouse single activity wheel chamber - CW. (C) Rat cage with a big and a small running wheel - RW. (D) Quantification of BrdU cell number confirmed that running increased hippocampal neurogenesis. BrdU cell number was significantly higher in the RW group than in the $S$ group $(*$ : $p<0.05)$. (E-G) Sample images of BrdU labeling in the DG in mice housed in S (E), CW (F) and RW cages (G). (H-J) Representative images of newborn GCs from mice housed in cages S, CW and RW, respectively. (K) New neurons did not differ significantly in total dendritic length between 
groups. (L) New neurons in the RW group had more dendritic branching points than those in the $\mathrm{S}$ group (*: $\mathrm{p}<0.05$ ). For $\mathrm{H}-\mathrm{I}, \mathrm{n}=35,30$ and 25 , respectively, for $\mathrm{S}, \mathrm{CW}$ and $\mathrm{RW}$. (M) Representative images of newborn GC dendritic processes in the outer molecular layer (2 each for S, CW and RW). (N) Total spine density of new neurons did not differ between groups in the outer molecular layer. (O) Mushroom spine density was significantly increased in both the $\mathrm{CW}$ and RW groups in the outer molecular layer $(*$ : $\mathrm{p}<0.01)$. For $\mathrm{N}-\mathrm{O}, \mathrm{n}=34,55$ and 48 for S, CW and RW, respectively. (P) Representative images of newborn GC dendritic processes in the middle molecular layer ( 2 each for $\mathrm{S}, \mathrm{CW}$ and $\mathrm{RW}$ ). (Q) Total spine density of new neurons did not differ between groups in the middle molecular layer. (R) Mushroom spine density was specifically increased in the RW group in the middle molecular layer (*: $\mathrm{p}<0.01$ ). For Q-R, $\mathrm{n}=38,54$ and 45, respectively for S, CW and RW. Scale bars: $100 \mu \mathrm{m}$ $(\mathrm{E}-\mathrm{G}), 50 \mu \mathrm{m}(\mathrm{H}-\mathrm{J})$ and $5 \mu \mathrm{m}(\mathrm{M}, \mathrm{P})$. 

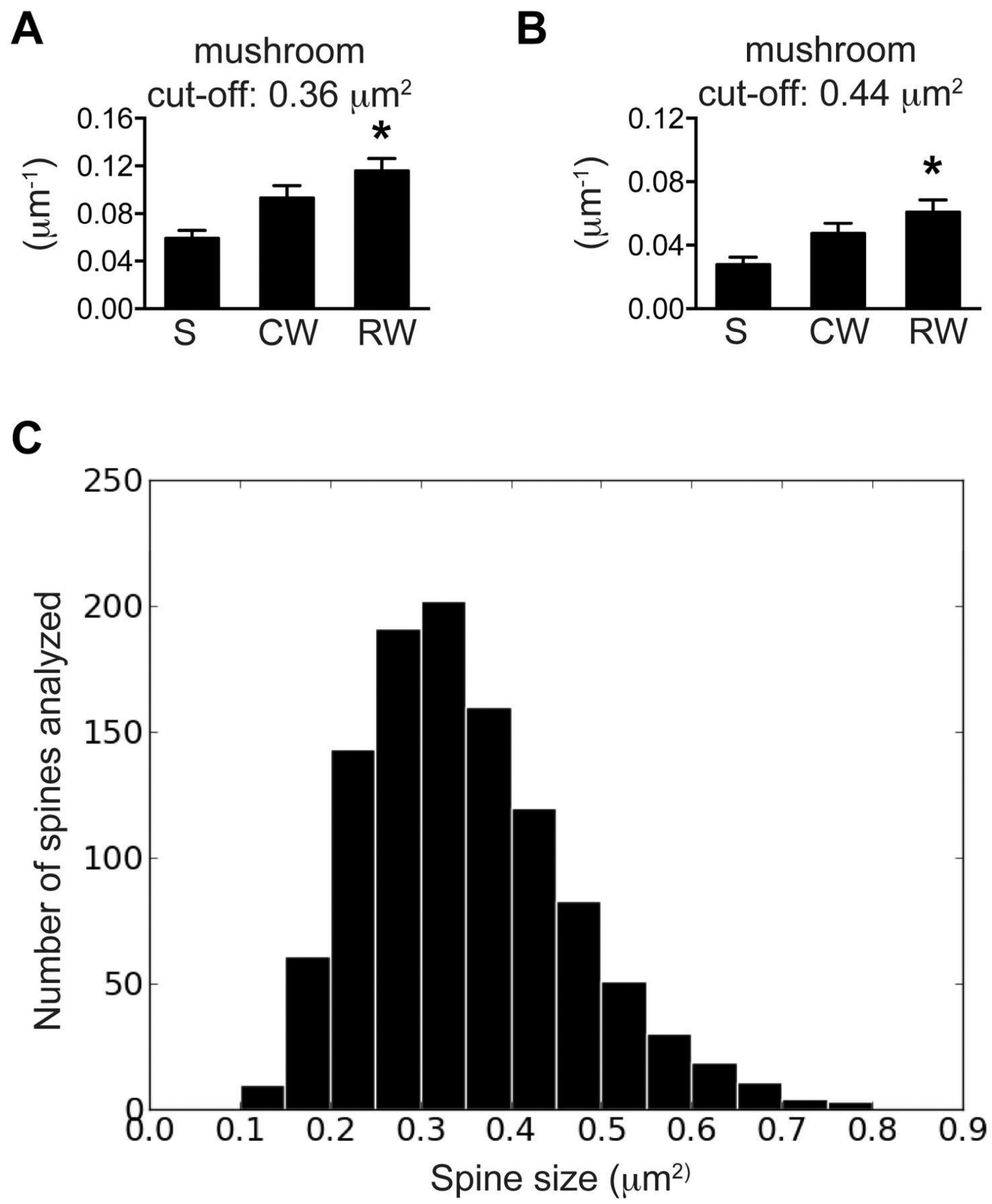

Figure 3. The differences between groups remained consistent when different threshold was set for the identification of mushroom spines

(A) The cut-off was set at $0.36 \mu \mathrm{m}^{2}$ for the identification of mushroom spines. (B) The cutoff was set at $0.44 \mu^{2}$ for the identification of mushroom spines. (C) The size distribution of all spines selected for tracing in Fig 2R. The Y-axes are the counts of spines for each bracket $\left(0-0.9 \mu \mathrm{m}^{2}\right.$, step: $\left.0.05 \mu \mathrm{m}^{2}\right)$. 
A
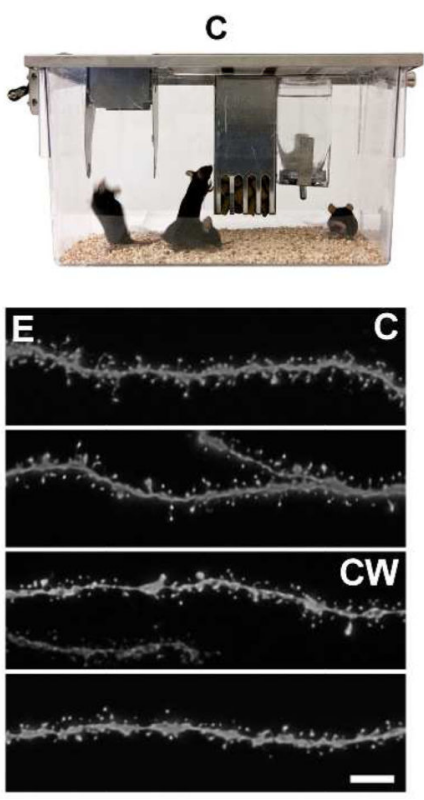

B
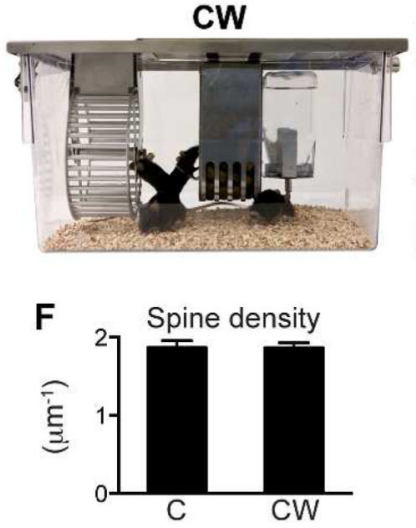

G

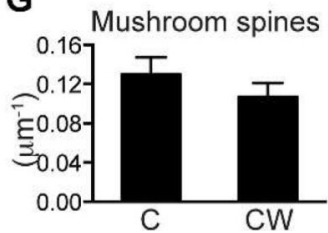

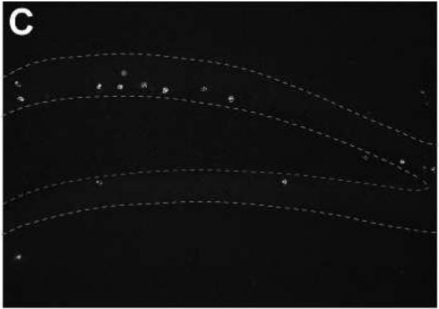
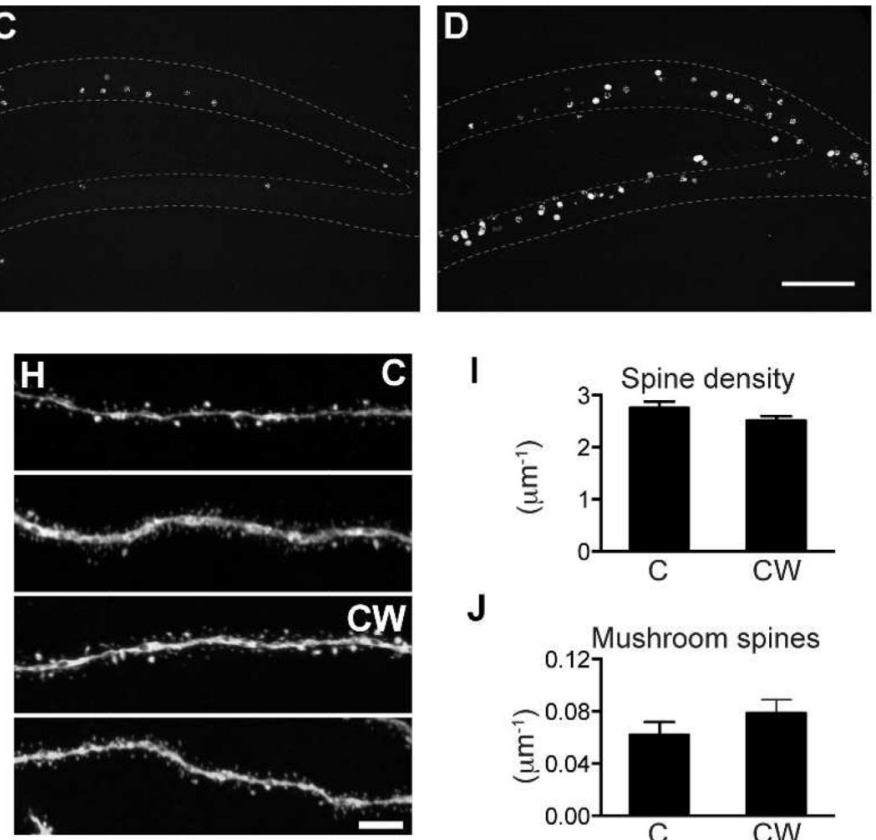

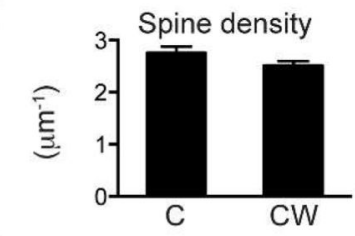

$J$

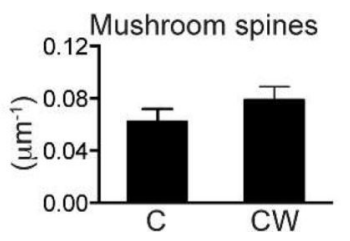

Figure 4. The running wheel in the $\mathrm{CW}$ cage did not affect spine maturation in newborn granule cells

(A) Control cage for the mouse single activity wheel chamber - C. (B) Mouse single activity wheel chamber - CW. (C) Sample image of BrdU labeling in mice housed in C cage. (D) Sample image of BrdU labeling in mice housed in CW cage. (E) Representative images of newborn GC dendritic segments in the outer molecular layer (2 each for C and CW). (F) Total spine density in the outer molecular layer. (G) Mushroom spine density in the outer molecular layer. For E-F, n=23 and 25 for $\mathrm{C}$ and CW, respectively. (H) Representative images of newborn GC dendritic segments in the middle molecular layer (2 each for $\mathrm{C}$ and CW). (I) Total spine density in the middle molecular layer. (J) Mushroom spine density in the middle molecular layer. For $\mathrm{G}-\mathrm{H}, \mathrm{n}=34$ and 36 for $\mathrm{C}$ and $\mathrm{CW}$, respectively. Scale bars: $100 \mu \mathrm{m}(\mathrm{C}-\mathrm{D})$ and $5 \mu \mathrm{m}(\mathrm{E}, \mathrm{H})$. 




B



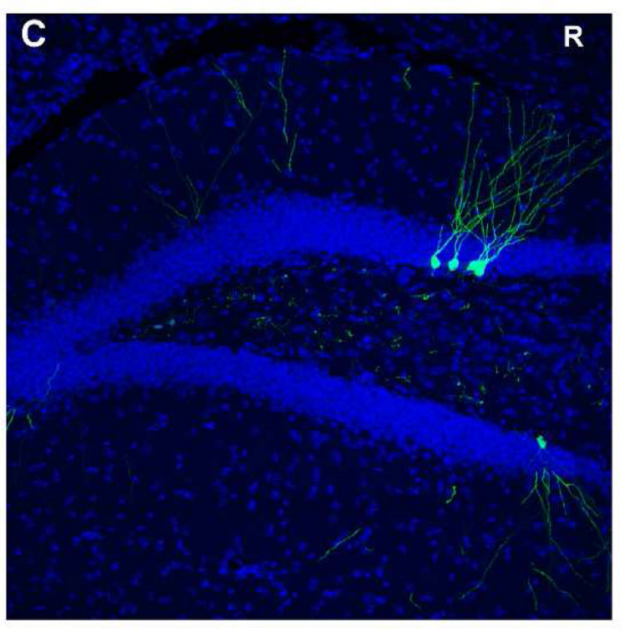
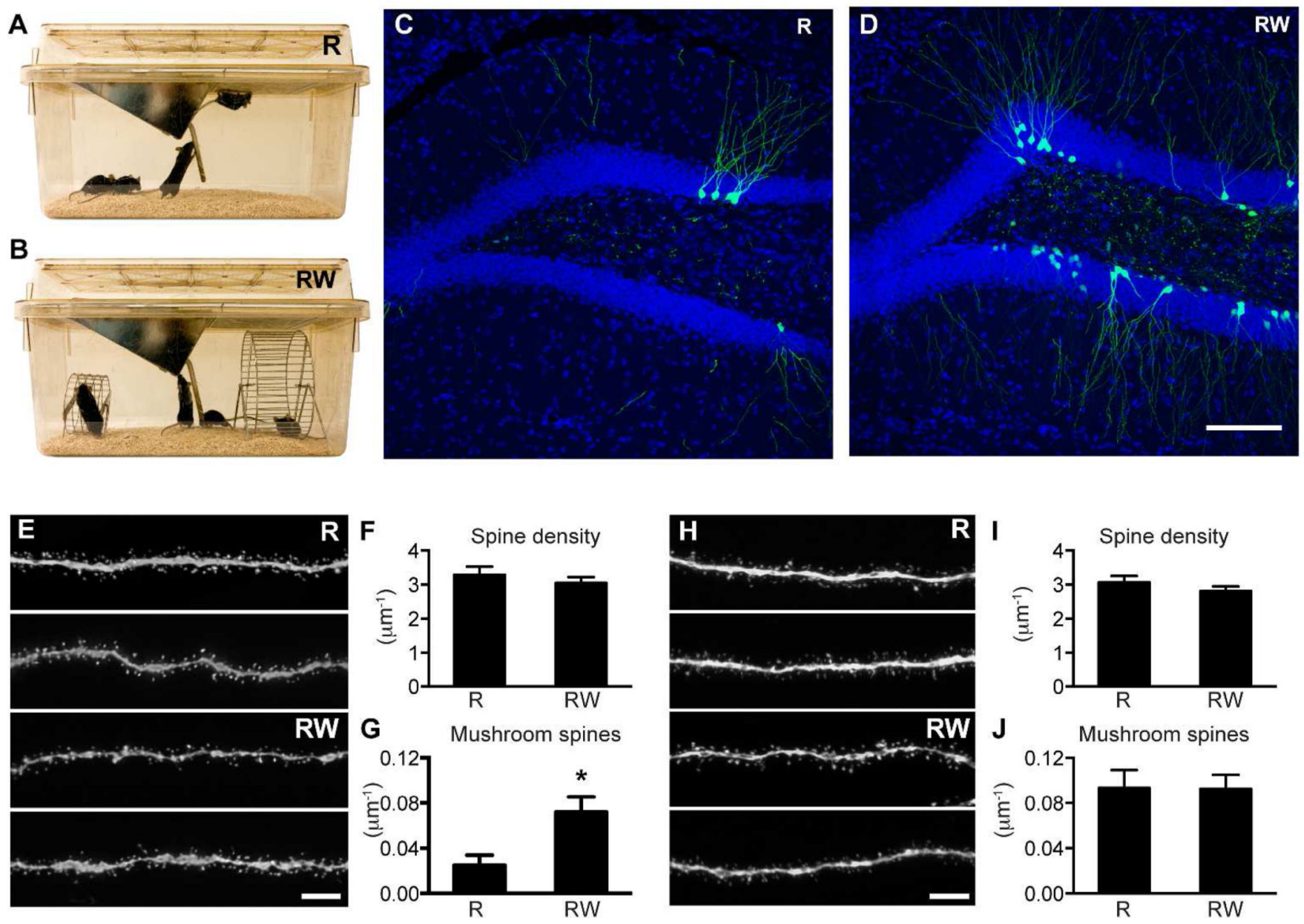

Figure 5. Inclusion of running wheels in the $R$ cage specifically promoted mushroom spine formation in the outer molecular layer

(A) Standard rat cage with no running wheel as a control - R. (B) Rat cage with running wheels - RW. (C-D) Sample images of GFP labeling in mice housed in R and RW cages, respectively. (E) Representative images of newborn GC dendritic segments in the outer molecular layer ( 2 each for R and RW). (F-G) Total and mushroom spine density in the outer molecular layer respectively (*: $\mathrm{p}<0.05$ in $\mathrm{F}$ ). $\mathrm{N}=31$ and 35 for $\mathrm{R}$ and $\mathrm{RW}$,

respectively. $(\mathrm{H})$ Representative images of newborn GC dendritic segments in the middle molecular layer ( 2 each for R and RW). (I-J) Total and mushroom spine density in the middle molecular layer, respectively. $\mathrm{N}=26$ and 34 for $\mathrm{R}$ and $\mathrm{RW}$, respectively. Scale bars: $100 \mu \mathrm{m}(\mathrm{C}-\mathrm{D})$ and $5 \mu \mathrm{m}(\mathrm{E}, \mathrm{H})$. 
A

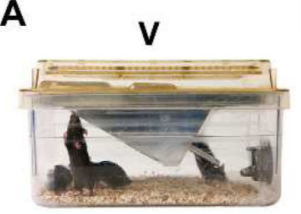

B
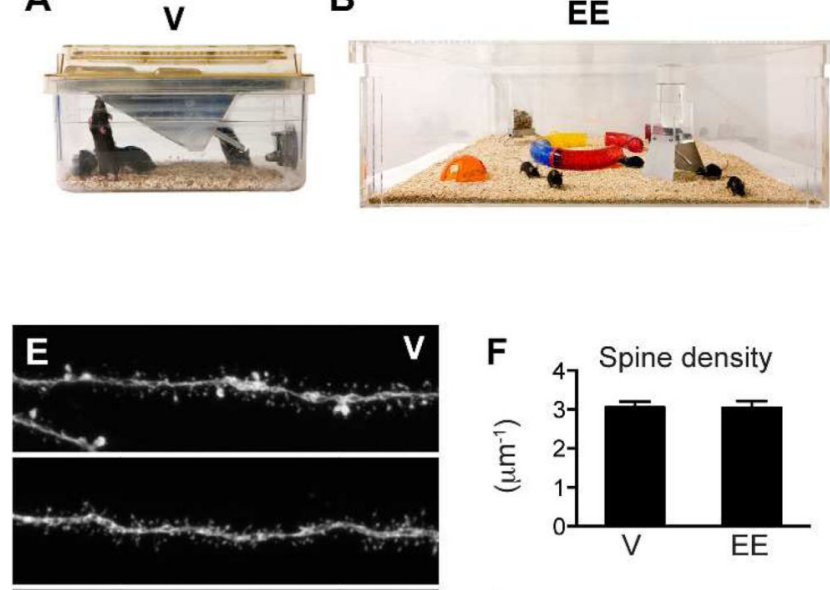

EE

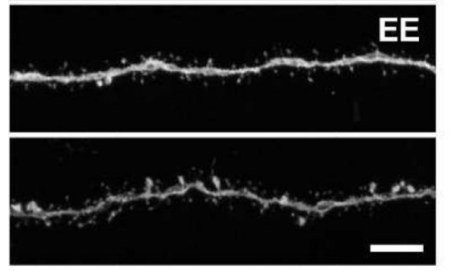

V

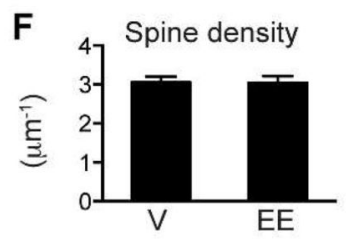

G Mushroom spines

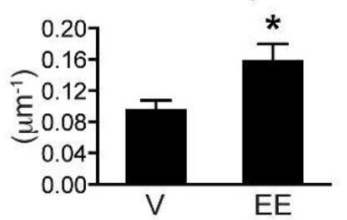

C
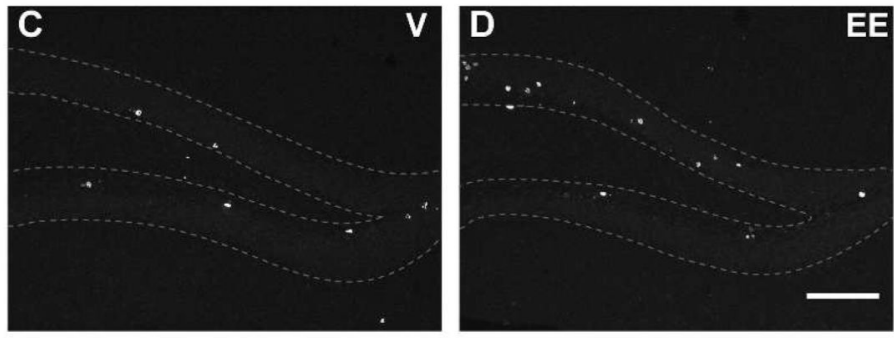

H
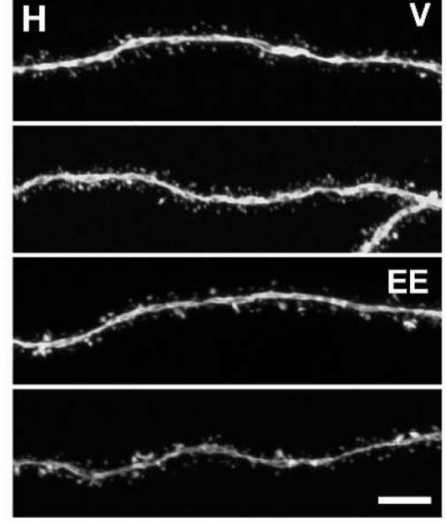

I



$\mathbf{J}$

Mushroom spines

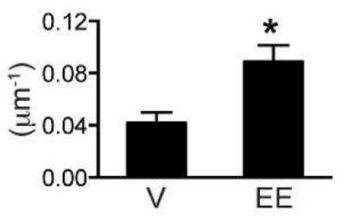

Figure 6. EE housing promoted mushroom spine formation in newborn granule cells

(A) Ventilated mouse cage - V. (B) Enriched environment cage -EE. (C-D) Sample images of BrdU labeling in mice housed in ventilated and EE cages. (E) Representative images of newborn GC dendritic segments in the outer molecular layer (2 each for V and EE). (F-G) Total spine density and mushroom spine density in the outer molecular layer. $\mathrm{N}=39$ and 27 for $\mathrm{V}$ and EE, respectively. (H) Representative images of newborn GC dendritic segments in the middle molecular layer ( 2 each for $\mathrm{V}$ and $\mathrm{EE}$ ). (I-J) Total spine density and mushroom spine density in the middle molecular layer. $\mathrm{N}=31$ and 32 for $\mathrm{V}$ and $\mathrm{EE}$, respectively. $\mathrm{EE}$ increased mushroom spine formation in both outer and middle molecular layers $(*, F$, $\mathrm{p}<0.05$ and $\mathrm{H}, \mathrm{p}<0.01)$. Scale bars: $100 \mu \mathrm{m}(\mathrm{C}-\mathrm{D})$ and $5 \mu \mathrm{m}(\mathrm{E}, \mathrm{H})$. 